\title{
LA SUCESIÓN DE ESTADOS ANTE LA CAIDA DEL BLOQUE SOCIALISTA: ELEMENTOS TEÓRICOS Y DESARROLLOS RECIENTES
}

\author{
Juan Miguel Ortega Terol
}

RESUMEN: En una perspectiva jurídica, el autor plantea en relación con una institución clásica en el Derecho internacional público, la sucesión de Estados, acerca del impacto que estas transformaciones estatales han tenido sobre la propia existencia del Estado y sobre diversos aspectos jurídicos que pueden resultar afectados por mor de dichas transformaciones.

\section{LA SUCESIÓN DE ESTADOS ANTE LA CAÍDA DEL BLOQUE SOCIALISTA: PLANTEAMIENTO DE LA CUESTIÓN}

Lejos de constituir entidades absolutamente inmutables, el devenir histórico confirma el carácter dinámico de la realidad estatal. Los Estados, como los seres vivos, dejando aparte una característica exclusiva de éstos, nacen, crecen - o disminuyen - y desaparecen. Frecuentemente, este tipo de fenómenos no

* Doutor pela UCLM. Profesor Titular de Derecho Internacional Público y Relaciones Internacionales Facultad de Ciencias Sociales. Cuenca. Universidad de Castilla-La Mancha.
RESUMO: Em uma perspectiva jurídica, o autor analisa uma instituição clássica do Direito internacional públio, qual seja, a sucessão de Estados, para discutir o impacto que estas transformações estatais tem sobre a própria existência do Estado e sobre diversos aspectos jurídicos que podem resultar afestados por conta de referidas transformações.

se producen aisladamente, sino más bien de forma sincopada, acompañando profundas transformaciones que tienen incidencia en múltiples planos de las relaciones internacionales. Podría señalarse, como hace Brigitte Stern, que este tipo de dinámicas se produce en forma de oleadas. Y, en esta dirección, la caída del bloque socialista ha ofrecido la más reciente de ellas. En efecto, el desvanecimiento de la escisión Este-Oeste se acompasó con una gran transformación territorial en el solar de lo que fuera la esfera de influencia soviética.

Lo que había sido la Unión Soviética acabó dando cobijo a quince Estados - y todavía permanecen enquistadas fuertes 
tensiones en Osetia y Abajzia (Georgia) y en Chechenia (Rusia) -. El que fuera Reino de los Serbios, Croatas y Eslovenos, la antigua Yugoslavia, quedó conformado en cinco Estados - seis teniendo en cuenta la reciente separación de Serbia y Montenegro -, y queda pendiente de resolver el estatuto de Kosovo. La República Checoslovaca se escindió en dos. Y la República Democrática Alemana se incorporó a la República Federal de Alemania.

Este conjunto de situaciones, en una perspectiva jurídica, ha planteado numerosas cuestiones en relación con una institución clásica en el Derecho internacional público: la sucesión de Estados. Y así, han surgido interrogantes acerca del impacto que estas transformaciones estatales han tenido, en primer lugar, sobre la propia existencia del Estado, esto es, si ante determinados cambios en los elementos constitutivos han continuado existiendo o no los Estado que los han sufrido, y en segundo lugar, sobre diversos aspectos jurídicos que pueden resultar afectados por mor de dichas transformaciones. De forma que podríamos preguntarnos si una determinada norma internacional sigue siendo oponible a un Estado que ha sufrido una transformación, qué bienes o qué deudas corresponden a un nuevo Estado o al Estado que incorpora un nuevo territorio; o cuál es la nacionalidad de los habitantes del territorio donde se producen los cambios, por citar algunas cuestiones más sobresalientes en este ámbito.

En el terreno de las acotaciones conceptuales conviene señalar que un primer interrogante que surge ante las transformaciones territoriales consistiría en dilucidar hasta qué punto los cambios afectan a la identidad del Estado o, de otra forma, se trataría de discernir si el Estado afectado continúa existiendo o si, por el contrario, ha desaparecido. Mientras que el conjunto de las situaciones que acompañan a la resolución de esa cuestión en el resto del territorio implicado en esos cambios, así como en el caso de llegar a una conclusión negativa a la primera cuestión se encuadrarían propiamente en el capítulo de la sucesión de Estados.

\section{LA CONTINUIDAD EN LA IDENTIDAD DEL ESTADO}

\subsection{La continuidad en la identidad del Estado}

Así pues, la primera cuestión a resolver consiste en saber si un Estado que sufre una transformación continúa o no existiendo o, de otra forma, si una alteración producida en una entidad estatal tiene como consecuencia su desaparición o, por el contrario, sigue siendo el mismo Estado existente con anterioridad a los acontecimientos histórico-políticos que producen un cambio en su estructura. Una $u$ otra opción tiene resultados diferentes desde el punto de vista jurídico: si se conviene que el Estado continúa existiendo, entonces seguirá vinculado por las obligaciones internacionales que le eran oponibles y disfrutando de sus derechos, excepto en la medida en que el cambio de circunstancias sea tal que haya que adecuarlos a la nueva situación ${ }^{1}$. Frente

\footnotetext{
${ }^{1}$ Dicho de otra forma, el sentido de la continuidad en la identidad sería el de preservar prima facie las relaciones jurídicas a pesar de los cambios en el sujeto de dichas relaciones; CRAWFORD, J., The creation of States in international law, Oxford, Clarendon Press, 1979, p. 400. Igualmente, en torno a estas cuestiones, ORTEGA TEROL, J.M., "Aspectos teóricos y prácticos de la continuidad en la identidad del Estado", ADI, vol. XV, 1999, pp. 273-323.
} 
a ello, si el Estado desaparece o ve reducida su extensión, su lugar será ocupado por otro Estado, constituido en sucesor del anterior $\mathrm{y}$, por tanto, como ya hemos señalado, los interrogantes planteados deberán resolverse por referencia a las reglas sobre sucesión de Estados.

\subsection{Las modificaciones de los elementos constitutivos del Estado}

Tradicionalmente, se alude a la existencia de un principio de continuidad en la identidad del Estado en las relaciones internacionales. Este principio se traduciría en la presunción de que un Estado continúa existiendo, a pesar de los cambios sufridos, mientras no pueda constatarse su desaparición. Sin embargo, ese principio juega un papel diferente según que la transformación afecte a unos u otros elementos constitutivos del Estado. No obstante, convendría destacar que en esta materia se manifiesta de forma especial la aspiración del Derecho Internacional por conseguir el máximo de estabilidad en las relaciones internacionales. Por ello, en el momento de efectuar una valoración de esa continuidad en la identidad, tendrá un peso específico, tanto el modo en que se produce la transformación estatal - es decir, si se trata de un proceso gradual, progresivo o pactado, o si es el resultado de una serie de acontecimientos traumáticos -, como el conjunto de intereses en disputa.

\subsubsection{Cambios en la organización política estatal}

Las transformaciones de la estructura política del Estado, por sí solas, no tienen incidencia sobre su posición en el panorama internacional. Dicho de otra forma, resulta indiferente que un Estado unitario pase a ser un Estado federal, que gobierne uno $u$ otro partido político e, incluso, que cambie de nombre. En este último sentido, por ejemplo, tendremos ocasión de comprobar como la Federación Rusa ha adoptado el papel de la Unión Soviética, o como a pesar de denominarse de similar forma la República Federativa de Yugoslavia no ha sido considerada continuadora de la República Federativa Socialista de Yugoslavia, o como Alemania es de hecho continuadora de la República Federal de Alemania.

En la práctica, los principales problemas se han suscitado como consecuencia de cambios no constitucionales en la forma de gobierno del Estado: golpes de Estado, procesos revolucionarios, etc. Por ejemplo, las autoridades soviéticas negaron cualquier continuidad con la Rusia de los zares, fundamentalmente, con la pretensión de desligarse de la deuda externa existente, lo que fue rechazado por las potencias occidentales.

Íntimamente ligada a esta cuestión encontramos el supuesto de cambios en los órganos de poder resultado de una ocupación extranjera. Este caso tendría relación con los límites al reconocimiento de nuevos Estados o de la anexión de nuevos territorios. Y, en efecto, la prohibición del uso de la fuerza impide considerar que un Estado pueda desaparecer como consecuencia de una ocupación bélica. Esta tesis ha sido la sostenida por las Repúblicas bálticas para afirmar su continuidad respecto de los Estados homónimos existentes hasta 1940, fecha de la ocupación soviética. Supuesto que en la doctrina anglosajona ha sido calificado como reversión, o dicho de otra forma, sería 
la recuperación de una identidad suspendida durante un período de tiempo.

\subsubsection{Cambios en la población del Estado}

La ausencia de exigencia alguna sobre el número de personas que deben poblar un territorio a efectos de estatalidad deja, en este punto, como única hipótesis lógico-formal que permita afirmar el desvanecimiento del Estado la desaparición total o casi total de su población, algo que resulta extremadamente difícil de asumir e imaginar actualmente.

\subsubsection{Las alteraciones territoriales}

Menos pacífica resulta la aplicación del principio de continuidad si la transformación estatal se produce en el elemento territorial. En algunos casos, las modificaciones territoriales no plantean dificultades sobre si el Estado que las sufre continúa existiendo o no. Así sucede cuando la pérdida o el crecimiento territorial se debe a causas naturales, cuando es el resultado de una cesión territorial por vía convencional o cuando se debe a la aplicación de una norma de Derecho Internacional o de Derecho interno. Por ejemplo, si Quebec llegara a separarse de Canadá por medios constitucionales, parece evidente que nadie dudaría de la continuidad del último.

Sin embargo, existen otros supuestos en los que resulta mucho más complicado establecer la existencia de continuidad o no del Estado preexistente a la alteración territorial. Los ejemplos que siguieron a la caída del muro de Berlín ilustran con profusión esta afirmación.

En primer lugar, la República checoslovaca se disolvió dando lugar a la República checa y a la República eslovaca, no considerándose a ninguna de ellas continuadora en la identidad de aquel Estado.

En segundo lugar, el desmembramiento de la Unión Soviética supuso que el territorio ocupado por un solo Estado pasara a serlo por $15 \mathrm{y}$, a pesar de la evidente alteración de su configuración territorial, Rusia fue considerada continuadora en la identidad de la Unión Soviética, manteniendo, entre otras cosas, la condición de miembro permanente del Consejo de Seguridad de las Naciones Unidas. En tercer lugar, la crisis yugoslava provocó la aparición de cinco Estados en el lugar anteriormente ocupado por la República Federativa Socialista de Yugoslavia (RFSY). Uno de ellos, la República Federativa de Yugoslavia, constituida por Serbia y Montenegro, pretendía erigirse en continuador de la RFSY, a lo que se opusieron, al menos en el momento inicial, Eslovenia, Croacia, Bosnia-Herzegovina y la Antigua República Yugoslava de Macedonia, junto a una gran parte de la Comunidad Internacional. ${ }^{2}$ Todavía en relación con este último proceso, no del

\footnotetext{
${ }^{2} \mathrm{El}$ caso de la antigua Yugoslavia ha resultado ser el más complejo de todos, convirtiéndose en un auténtico galimatías jurídico que encuentra derivaciones en el momento actual, a pesar de que la República Federativa de Yugoslavia es miembro de Naciones Unidas desde el 1 de noviembre de 2000. En este sentido resulta sumamente ilustrativa la solicitud de revisión de la sentencia de 11 de julio de 1996 de la CIJ planteada por Serbia y Montenegro (Véase CIJ, Sentencia de 3 de febrero de 2003) o las amplias consideraciones sobre la cuestión realizadas por la propia Corte en su sentencia, de 26 de febrero de 2007, en el asunto relativo a la aplicación de la Convención sobre prevención y sanción del delito de genocidio (Bosnia y Herzegovina contra Serbia y Montenegro). Una buena muestra de estas afirmaciones puede verse, muy particularmente, en los parágrafos 88 a 99 de la última resolución citada. Ambas pueden encontrarse en http://www.icj-cij.org.
} 
todo finalizado, habría que señalar que la separación de Montenegro de Serbia, tras el referéndum celebrado el 21 de mayo de 2006, ha dejado intacta la identidad del segundo Estado que mantiene su posición en Naciones Unidas y en relación con los tratados multilaterales depositados ante el Secretario General de Naciones Unidas, mientras que el primero solicitó su admisión como nuevo miembro de la ONU - y la obtuvo, en junio de ese año. ${ }^{3}$ Finalmente, la República Democrática Alemana desapareció absorbida por la República Federal de Alemania.

En todos estos casos, se produjo un cambio considerable en las dimensiones territoriales de los Estados afectados, sin que aquel principio de continuidad haya jugado el mismo papel. Se puede afirmar que, ante este tipo de situaciones, el Derecho Internacional no ha elaborado criterios claros y objetivos relativos a la continuidad en la identidad ${ }^{4}$. Esta calificación - la de Estado continuadores más bien el resultado de un proceso, de mayor o menor duración en el tiempo, en el que se tienen en cuenta diversos factores. Por una parte, en los dos supuestos en que es constatable la desaparición de un Estado, checoslovaco y alemán, no se han producido grandes problemas debido a la estabilidad demostrada en sendos procesos pacíficos, siendo incluso calificado el primero de ellos

\footnotetext{
${ }^{3}$ También en relación con esta cuestión resulta de interés la Sentencia de la CIJ, de 26 de febrero de 2007 , citada en la nota anterior.

${ }^{4}$ CZAPLINSKI, W., «La continuité, l'identité et la succession d'Etats - Evaluation de cas récents», RBDI, 1993/2, p. 379; VERHOEVEN, J., «La reconnaissance internationale: déclin ou renouveau?», AFDI, vol.XXXIX, 1993, p. 33.
}

como «divorcio ejemplar». ${ }^{5}$ Por otra parte, sin embargo, los acontecimientos que llevaron a los desmembramientos de la Unión Soviética y de Yugoslavia presagiaban un resultado muy distinto. En ambos casos, la continuidad en la identidad de los Estados preexistentes fue puesta en duda y ha sido saldada de diferente manera. En este sentido, se pueden extraer una serie de criterios de la práctica estatal para hacer frente a estos problemas.

\subsection{Criterios para valorar la existencia de la continuidad en la identidad del Estado}

No obstante esa ausencia de criterios objetivos para determinar cuándo, ante una gran transformación territorial, un Estado continúa existiendo o, por el contrario, desaparece, la práctica de los Estados proporciona una serie de datos que permiten efectuar una valoración de la cuestión.

\subsubsection{La pretensión de continuidad en la identidad}

En primer lugar, para determinar si un Estado continúa en la identidad del Estado que sufre la transformación territorial, juega un papel fundamental el hecho de que aquél se considere a sí mismo continuador de éste y la pretensión de que los demás lo reconozcan como tal. En el ámbito geográfico que venimos analizando podría señalarse que se reclamaron continuadores Rusia respecto de la Unión Soviética y la RFY respecto de la

${ }^{5}$ ANDRES SAENZ DE SANTA MARIA, M.P., «Problemas actuales de la sucesión de Estados», Cursos de Derecho Internacional de Vitoria-Gasteiz, 1993, p. 194. 
RFSY. Parecida consideración cabría hacer en relación con Alemania en la medida en que la Ley Fundamental de Bonn de la RFA ya contemplaba la posibilidad de incorporación de los Lander de la RDA. Y asimismo esa es la posición mantenida por Serbia tras la definitiva dislocación de las dos últimas repúblicas yugoslavas que quedaban ligadas. Por el contrario, ni la República Checa, ni Eslovaquia reclamaron tal condición.

\subsubsection{La actitud de los Estados especialmente interesados}

Como hemos señalado, la duda sobre la continuidad de un Estado proviene de una transformación territorial que, en la mayoría de los casos, producirá el nacimiento de nuevos Estados. La posición de estos Estados en relación con la anterior pretensión será fundamental en el momento de resolver si existe o no dicha continuidad. A modo de ejemplo, puede contrastarse la diferente posición de los países ex-soviéticos y la de los países ex-yugoslavos y la incidencia sobre las respectivas pretensiones de la Federación Rusa y la RFY.

\subsubsection{La actuación de terceros Estados}

La postura adoptada por el resto de la Comunidad internacional que, en unos casos, se manifestará mediante la aceptación tácita o, en otros, mediante el reconocimiento expreso de la pretensión de continuidad, será determinante para el alcance y eficacia de la misma. En esta dirección, habría dos índices especialmente relevantes de esa posición: por una parte, la continuidad del status como miembro de organizaciones internacionales, eludiendo el mecanismo que los actos constitutivos de éstas prevean para adquirir tal condición. Así, es sabido que Rusia ocupó el puesto de la URSS en Naciones Unidas, incluyendo el muy privilegiado estatuto de miembro permanente del Consejo de Seguridad. Mientras que la posición de la RFSY dio lugar a un auténtico enredo político-jurídico, al mantenerse el puesto y rúbrica de Yugoslavia en la Asamblea General, así como su bandera en el patio de la sede de la ONU, pero exigiendo a la RFY su solicitud de admisión como nuevo miembro. Igualmente, podría señalarse que Alemania no tuvo que iniciar el procedimiento de adhesión a las Comunidades Europeas. Por otra parte, más recientemente, la continuidad de Serbia se ha saldado sin controversias.

En definitiva, carecemos de una lista de indicios objetivos que nos permitan afirmar cuándo, ante una crisis territorial de un Estado, puede afirmarse rotundamente que dicho Estado continúa existiendo y será la conjunción de diversos factores subjetivos los que permitirán alcanzar dicha conclusión. El corolario lógico de una respuesta afirmativa tras un proceso de estas características será el mantenimiento de los derechos y obligaciones respecto del Estado cuya identidad se reclama continuada.

No obstante, conviene retener los datos esbozados hasta el momento, puesto que como tendremos ocasión de comprobar la resolución de las dificultades planteadas tendrá consecuencias en la segunda parte de nuestro análisis: la sucesión de Estados.

\section{LA SUCESIÓN DE ESTADOS}

Y es que las transformaciones del territorio de un Estado que provocan las dudas sobre su continuidad generan otra serie de problemas. 
Normalmente, si un Estado desaparece $o$, aunque continúe existiendo, deja de ejercer competencias sobre un determinado territorio, su lugar es ocupado por otro Estado, bien constituyéndose un nuevo Estado, bien mediante la ampliación de la base territorial de un Estado preexistente. A este hecho se le llama sucesión de Estados. ${ }^{6}$ Son muy diversos los interrogantes que surgen de una sucesión de Estados. Así, entre otros, podemos preguntarnos si es vinculante para el Estado que ocupa el lugar del predecesor un tratado que, antes de ese hecho, era aplicable al territorio donde se produce la sustitución; o cuál es la nacionalidad de los habitantes del territorio, ¿la del Estado sucesor o la del predecesor?; o a quién corresponde la titularidad de los bienes del Estado predecesor situados en el territorio donde se produce la sucesión. En cualquier caso, conviene subrayar que, a pesar de la terminología utilizada, el nombre de esta institución no prejuzga el resultado de las diversas cuestiones que plantea este fenómeno. Dicho de otro modo, si la sucesión de Derecho privado prevé la asunción de derechos y de determinadas obligaciones del causahabiente por parte del sucesor, la sustitución de un Estado por otro en un determinado territorio no supone necesariamente que éste se vea gravado con las obligaciones o beneficiado por los derechos atribuibles al Estado

\footnotetext{
${ }^{6}$ Existen numerosas definiciones de la sucesión de Estados. Las Convenciones de Viena, que estudiaremos más adelante, la definen como «la sustitución de un Estado por otro en la responsabilidad de las relaciones internacionales de un territorio». Por otra parte, para O'CONNELL se trata de la «transferencia de territorio de una comunidad nacional a otra»; The Law of State succession, 1956, Cambridge University Press, p. 1.
}

predecesor. Empero, aunque equívoca en su significación jurídica, resulta gráfica en cuanto a los problemas que se pretenden resolver, como ha señalado el Prof. Blanc Altemir, se trata de saber qué pasa con la "herencia" del Estado predecesor: con su patrimonio jurídico y material. ${ }^{7}$

Antes de introducirnos en los vericuetos teóricos del tema, conviene poner de manifiesto que, como institución clásica de la teoría de los sujetos en Derecho internacional, esta cuestión ha merecido la atención de una doctrina que se ha mostrado muy tozuda a la hora de someter la práctica de los Estados en la materia a soluciones prefiguradas especialmente la doctrina anglosajona y francófona -, como demuestra el hecho de que, a pesar del escaso éxito obtenido por la labor de codificación al respecto, la CDI haya seguido obstinada hasta tiempo reciente en dar soluciones deudoras de los trabajos que cristalizaron en las escasamente eficaces Convenciones de Viena o, del mismo modo, que la ILA o el IDI sigan estudiando estas cuestiones desde parámetros similares. ${ }^{8}$

${ }^{7}$ BLANC ALTEMIR, A. La Herencia Soviética ( La Comunidad de Estados Independientes y los problemas sucesorios). Madrid: Tecnos, 2004.

${ }^{8}$ No obstante, conviene poner de manifiesto la mayor disposición de estas dos prestigiosas Instituciones a abordar estas cuestiones de una forma más realista, adecuando sus conclusiones a la práctica más reciente en la materia. Pueden verse, por ejemplo, los más recientes trabajos de la ILA, en su septuagésimo segunda conferencia, celebrada en Toronto, en junio de 2006, con el título de "Aspects of the Law of State sucesión (Economic Aspects of State Sucesión)", en Report of the 76th Conference of the ILA, pp.301-336. Respecto del IDI damos cuenta de los últimos desarrollos realizados en ORTEGA TEROL, J.M., "La sucesión de Estados en materia de bienes, archivos y deudas de Estado: la puesta a prueba de un ejercicio codificador", REDI, vol. LIV, 2002-1, pp.131-142. 


\subsection{Las Convenciones de Viena sobre sucesión de Estados}

En efecto, la obra codificadora de las Naciones Unidas cuenta con dos instrumentos pertinentes en este ámbito: la Convención de Viena, de 23 de agosto de 1978, sobre la sucesión de Estados en materia de tratados internacionales y la Convención de Viena, de 8 de abril de 1983, sobre la sucesión de Estados en materia de bienes, archivos y deudas de Estado. Sólo la primera está en vigor, si bien únicamente cuenta con veinte Estados parte, mientras que la segunda, que no está vigente, cuenta sólo con siete Estados parte, y ello veintiocho y veintitres años, respectivamente, después de la celebración de las Conferencias de codificación. ${ }^{9}$ A nuestro juicio, este dato resulta suficientemente demostrativo de la escasa aceptación que ambas Convenciones han tenido entre los Estados.

Asimismo, incluso para los Estados parte en la Convención de 1978, se plantean una serie de obstáculos técnico-jurídicos que limitan considerablemente la utilidad de aquéllas en la solución de los problemas para los que fueron diseñadas. Así, la aplicación de

${ }^{9}$ La propia entrada en vigor de la Convención de 1978 ya plantea algunos problemas en relación con la solución de los problemas para los que fue diseñada. Así, entre las 15 ratificaciones necesarias para su vigencia (actualmente cuenta con 20 Estados partes), se contaba la de Yugoslavia, un Estado que, a juicio del Consejo de Seguridad, de la Comisión de Arbitraje para Yugoslavia y numerosos Estados, dejó de existir antes de que se produjera dicha entrada en vigor. Por otra parte, resulta destacable el hecho de que los otros cuatro Estados ex-yugoslavos hayan utilizado el mecanismo de la notificación de sucesión para acceder a la misma, haciendo uso de una técnica que, como veremos, la Convención prevé para un supuesto de hecho teóricamente distinto. las Convenciones no es retroactiva, excepto para el Estado sucesor que declare que aplicará sus disposiciones a su propia sucesión de Estados, producida antes de la entrada en vigor de cualquiera de ellas ${ }^{10}$. Además, hay un problema lógico en los acontecimientos que provocan una sucesión de Estados que genera dificultades a esa aplicación de las disposiciones de las Convenciones, puesto que, aunque el Estado predecesor sea parte en las mismas, los nuevos Estados sucesores que surjan de una sucesión que le afecte no lo serán y, por tanto, sólo les serán aplicables en la medida en que incorporen normas de Derecho Internacional general.

Por ello, no deja de resultar curioso que la CDI emprendiera el análisis de otro capítulo de la cuestión: la nacionalidad de las personas naturales en relación con la sucesión de Estados. Y, no obstante, dados los precedentes, no es tan extraño que el resultado de su trabajo esta vez no haya derivado en una conferencia de codificación, ni en la forma de convención, y el proyecto de artículos en la materia, de 1999, fue endosado en una resolución de la Asamblea General, en 2001, en forma de declaración con la consideración de que pueden ser "una guía útil".

Sin embargo, a pesar de todas estas consideraciones, existe un alto grado de consenso doctrinal acerca de algunas disposiciones de ambas Convenciones que son consideradas reflejo del Derecho

${ }^{10}$ Art. 7 de la Convención de Viena sobre sucesión de Estados en materia de tratados internacionales y art. 4 de la Convención de Viena sobre sucesión de Estados en materia de bienes, archivos y deudas de Estado. Esta posibilidad únicamente ha sido utilizada hasta la fecha por Eslovaquia en relación con el Convenio de 1978. 
consuetudinario en este ámbito. En cambio, respecto de otras disposiciones, la práctica de los Estados muestra un claro desacuerdo con lo establecido en ellas. A ello vamos a dedicar nuestra atención a partir de ahora, siguiendo un esquema que resulta poco habitual en los manuales al uso, pero que se ajusta a la visión propuesta en el título de nuestra intervención.

\subsection{Tipología sucesoria}

Así pues, al analizar las consecuencias que pueden derivarse de una sucesión de Estados hay que considerar, en primer lugar, que no todos los hechos sucesorios son iguales o, dicho de otra forma, que existen diferentes tipos de sucesión, respecto de los cuales pueden predicarse soluciones distintas. Así, una sucesión de Estados puede producirse de las siguientes formas:

a) por una unificación de Estados, esto es, dos o más Estados se unen para constituir un nuevo Estado. Puede citarse como ejemplo el de Yemen unificado, resultado de la unión de Yemen del Norte y Yemen del Sur.

b) por la absorción de un Estado por otro. En este caso, un Estado desaparece para integrarse en otro Estado. A pesar de la terminología habitualmente utilizada, la llamada «reunificación» alemana constituiría un supuesto de este tipo. Se puede señalar el hecho de que las Convenciones de Viena sobre sucesión de Estados no consideran esta posibilidad de forma independiente a la unificación de Estados, siendo precisamente la integración de la República Democrática de Alemania en la República Federal de Alemania, la que ha puesto de manifiesto algunas discrepancias de los regímenes previstos por aquéllas con la práctica de los Estados.

c) por la transferencia de parte del territorio de un Estado a otro Estado. Se trataría de un supuesto de cesión territorial, sumamente raro en la actualidad, por el que un Estado traspasa a otro Estado una parte de su territorio. En perspectiva histórica, cabría señalar el caso de Alsacia y Lorena, que sucesivamente fueron parte de Alemania y de Francia, o la cesión a título oneroso de Alaska a Estados Unidos por parte de Rusia.

d) por el surgimiento de un nuevo Estado como consecuencia de la descolonización de un territorio. Realmente, se trata de un supuesto que, por la propia dinámica de la que trae causa, se encuentra abocado al agotamiento; aunque pueden citarse algunos casos recientes como el de los Estados federados de Micronesia o el de Timor. Los Estados constituidos de esta forma reciben el nombre de Estados de reciente independencia y las Convenciones de Viena preveían para ellos un régimen claramente favorable. Tras unas primeras controversias doctrinales acerca de su adecuación a la práctica de los Estados, parece que actualmente existe un amplio acuerdo acerca del alcance general de la regulación para este tipo de supuestos. Por otra parte, la atención prestada por la Comisión de Derecho Internacional a estos casos llevó a una mayor precisión de las reglas previstas para los mismos, lo que ha supuesto que en algunos de 
los acontecimientos más próximos hayan servido a los nuevos Estados en su aproximación a las cuestiones que estamos tratando.

e) por el nacimiento de nuevos Estados en el territorio donde antes ejercía sus competencias uno sólo. No obstante, lejos de constituir una categoría única, la aparición de un nuevo Estado en el territorio de un Estado preexistente puede revestir formas diversas que den lugar a comportamientos distintos frente al hecho sucesorio. Por una parte, la Convención de 1978 establecía un tipo único para estos supuestos, mientras que la Convención de 1983 se refería a dos: separación de parte o partes del territorio de un Estado para formar dos o más nuevos Estados, donde el Estado predecesor continúa existiendo, y disolución de un Estado, donde el Estado predecesor desaparece. Sin embargo, como hemos tenido ocasión de comprobar al referirnos los casos de la URSS o de Yugoslavia, las dificultades para determinar, en los primeros momentos de los acontecimientos que dan lugar a la sucesión, si existe o no un Estado continuador desaconsejan esta aproximación, es decir, no parece apropiado hacer reposar una determinada clasificación de supuestos sobre la existencia o no de un Estado continuador.

Por otra parte, la práctica muestra cómo los Estados se comportan de forma diferente según el modo en que se produzca la sucesión, esto es, si es el resultado de un proceso progresivo o pactado-lo que, adoptando la terminología de la Convención de 1983, llamaremos disolución de Estados - o si es el resultado de una serie de acontecimientos traumáticos, más notables si existe una situación abiertamente violenta lo que denominaremos desmembramiento de Estados -.

Antes de analizar las soluciones en los diferentes ámbitos materiales para cada uno de estos supuestos, conviene realizar una última precisión: la prohibición del uso de la fuerza en las relaciones internacionales impide que se establezca una relación sucesoria mediando aquél. Esto tiene una incidencia clara en algunos de los supuestos anteriores que sólo pueden tener lugar de una forma pacífica. Así ocurre en las tres primeras categorías: unificación, absorción de un Estado por otro y transferencia territorial. Sin embargo, en el resto de casos, como ocurre en el desmembramiento de Estados, las circunstancias que dan lugar al surgimiento de nuevos Estados pueden entrañar episodios violentos, si bien no aparece en ellos la participación de un Estado tercero, por lo que no puede hablarse en sentido estricto de fuerza prohibida por el Derecho Internacional. Baste citar el intento de golpe de Estado en la Unión Soviética, con una incidencia fundamental en los posteriores acontecimientos, o la guerra fraticida iniciada en Yugoslavia.

\subsection{Sucesión de Estados y ordenamiento jurídico internacional.}

Una de las primeras cuestiones que se plantea ante una sucesión de Estados es la de si los Estados sucesores están vinculados por las normas internacionales aplicables al territorio con anterioridad a la sucesión. Dicho de otra forma, si un Estado se encuentra sujeto a una serie de normas, cuando deja de ejercer competencias sobre un determinado 
territorio, ¿son aplicables dichas normas al Estado que ocupa su lugar?

Las respuestas, según el supuesto de que se trate, varían en torno a dos polos opuestos: a) principio de continuidad, los Estados sucesores seguirán vinculados por las obligaciones contraidas y los derechos disfrutados por el Estado predecesor; y b) principio de la «tabla rasa», los Estados sucesores no están vinculados por el conjunto de derechos y obligaciones atribuible al Estado predecesor. No obstante, es preciso señalar que ni uno ni otro principio encuentran una aplicación radical en la práctica de los Estados.

Por una parte, en los casos en que se aplica el primer principio, la posición del Estado sucesor no se traduce automáticamente en la continuidad de todos los tratados celebrados por el Estado predecesor. Así, los llamados tratados «cerrados»o de participación restringida no se someterán necesariamente a esa regla. Otro tanto cabe decir de tratados aplicables solamente respecto de una parte del territorio del Estado predecesor. Ilustrémoslo con un ejemplo: la República Checoslovaca había celebrado un acuerdo con Hungría para la construcción de un complejo hidráulico en la frontera entre ambos países. Tras la disolución del primer Estado, la zona en que debía construirse la presa quedó bajo soberanía eslovaca, que fue la que asumió las obligaciones y los derechos derivados de aquel tratado, siendo aceptada esta postura por las autoridades húngaras. ${ }^{11}$

${ }^{11}$ Vid. CIJ, Sentencia de 25 de septiembre de 1997, Asunto relativo al Proyecto Gabcíkovo-Nagymaros, Hungría contra Eslovaquia.
Por otra parte, conviene subrayar que la «tabla rasa» no supone una total ruptura con el entramado jurídico del predecesor. En primer lugar, porque los Estados a los que se aplica este principio pueden optar por la continuidad de algunos de los vínculos jurídicos contraidos por el Estado predecesor, a través del mecanismo de la notificación de sucesión. En segundo lugar, porque existirían algunas, pocas, reglas de alcance general que supondrían la obligación de seguir cumpliendo determinadas exigencias por los Estados sucesores independientemente de la forma en que tuviera lugar la sucesión de Estados. Así, las relativas al respeto de las fronteras internacionales preexistentes o las de los llamados regímenes territoriales objetivos. ${ }^{12}$

\subsubsection{Sucesión de Estados y costumbre}

El interrogante que se plantea en este punto es si una regla consuetudinaria vincula a los nuevos Estados surgidos de una sucesión. ${ }^{13}$ La práctica reciente muestra cómo se ha exigido de manera insistente a los nuevos Estados el respeto de una serie

${ }^{12}$ Vid. arts. 11 y 12 de la Convención de Viena de 1978 sobre sucesión de Estados en materia de tratados internacionales. Asimismo, en el primer sentido, puede verse la Sentencia de la CIJ, de 3 de febrero de 1994, asunto relativo a la diferencia territorial entre la Jamahiriya Arabe Libia y Chad y, en el segundo, la ya citada en el asunto del Proyecto Gabcíkovo-Nagymaros.

${ }^{13}$ La cuestión guarda una íntima relación con la participación en el proceso de formación de normas consuetudinarias o, dicho de otra forma, si una regla de derecho consuetudinario es oponible a un Estado que no haya participado en su proceso de formación. Al respecto, puede verse FERNANDEZ TOMAS, A.; SANCHEZ LEGIDO, A.; y ORTEGA TEROL, J.M., Manual de Derecho Internacional Público, pp.93 y ss. 
de normas de las que se puede presumir ese carácter, fundamentalmente, en relación con el reconocimiento de los derechos humanos, la prohibición del uso de la fuerza en las relaciones internacionales, la obligación de solucionar pacíficamente las diferencias y el respeto de las fronteras internacionales. No obstante, en sentido contrario, convendría hacer referencia al importante movimiento contra las estructuras económicas existentes iniciado por los países surgidos de la descolonización y que supuso el cuestionamiento de un conjunto de reglas consideradas de alcance general por la mayoría de los Estados occidentales. En cualquier caso, la mayor parte de la doctrina parece inclinarse por una respuesta inicialmente afirmativa a la cuestión planteada, si bien niega el carácter general a supuestas normas consuetudinarias que encuentren la oposición de un grupo significativo de Estados.

\subsubsection{Sucesión de Estados en materia de tratados internacionales}

Frente a una norma consuetudinaria general, de la que puede decirse que obliga a todos los Estados que no se opusieran a su formación, un tratado internacional, como tal, requiere del consentimiento de los Estados para que sus disposiciones les sean aplicables. En este sentido, el problema ante una sucesión de Estados es discernir si los tratados celebrados por el Estado predecesor continúan vinculando al Estado o Estados sucesores. A esta cuestión se dedica la Convención de Viena sobre sucesión de Estados en materia de tratados internacionales que, como vimos, plantea numerosos inconvenientes en cuanto a su alcance. Así, se puede observar que la práctica internacional en unas ocasiones se acomoda a lo previsto en la Convención de 1978 y en otras no, según la forma que revista la sucesión de Estados.

En primer lugar, respecto de los fenómenos de unificación de Estados, la Convención de Viena se inclina por la aplicación del principio de continuidad, esto es, los tratados celebrados por los Estados predecesores seguirán vinculando al Estado sucesor resultante de su unión, salvo que se convenga otra cosa con el resto de Estados partes o que resulte incompatible con el objeto y fin del tratado de que se trate. No obstante, esos tratados se aplicarán sólo respecto de la parte del territorio del Estado sucesor respecto de la cual estuvieran en vigor, a menos que el Estado sucesor notifique o convenga con las demás partes otra cosa, según el caso. Efectivamente, ésta parece haber sido la línea seguida en el caso de Yemen. Sin embargo, un supuesto reciente que, teóricamente, debía incluirse en esta categoría de la Convención ha seguido un camino distinto. Nos referimos a la llamada «reunificación» alemana.

Como tuvimos ocasión de señalar, el caso alemán podía ser calificado más adecuadamente como absorción de un Estado por otro. En efecto, lo que se produjo tras la caída del muro de Berlín fue la desaparición de la República Democrática de Alemania y la incorporación de su territorio en la República Federal de Alemania, cuyas estructuras ocuparon el lugar de las correspondientes al régimen anterior. En este contexto, la solución general adoptada respecto de los tratados internacionales celebrados por los Estados predecesores fue la de la continuación de los que vinculaban a la RFA, que mantuvo su condición de miembro de las Organizaciones 
Internacionales a las que pertenecía, incluida la $\mathrm{CE}$, haciendo extensible su aplicación al territorio de la RDA, y, frente a una inicial posición en el sentido de estudiar los suscritos por la RDA, finalmente se optó por la terminación de la mayoría de los mismos.

Curiosamente, en tercer lugar, el ejemplo alemán se aproxima enormemente a lo previsto en la Convención de Viena para los supuestos de sucesión respecto de una parte de territorio, lo que hemos denominado transferencia territorial, que se regirían por la denominada regla de la movilidad del ámbito geográfico de aplicación de los tratados. Dicho de otra forma, los tratados del Estado predecesor dejarán de estar en vigor respecto del territorio objeto de la sucesión y, en su lugar, pasarán a estar en vigor los del Estado sucesor, con las salvedades que puedan derivarse de la existencia de tratados localizados.

La cuarta categoría a la que hemos hecho referencia es la del surgimiento de nuevos Estados como consecuencia de la descolonización de un territorio. Los Estados surgidos de esta manera reciben el nombre de Estados de reciente independencia. La Convención de 1978 establece para ellos un régimen enormemente flexible que ha sido bautizado metafóricamente como tabla rasa matizada y supone que esos Estados no están obligados a mantener en vigor o a pasar a ser partes en un tratado que estuviera en vigor respecto del territorio de que se trate en la fecha de la sucesión de Estados. No obstante, los Estados de reciente independencia podrán devenir partes en un tratado multilateral que estuviera en vigor respecto de su territorio mediante una notificación de sucesión, salvo que se trate de un acuerdo de participación restringida o sea incompatible con el objeto y el fin del tratado. Asimismo, como señalamos anteriormente, también se verán obligados por las disposiciones relativas a regímenes de fronteras y otros regímenes territoriales.

Respecto de los tratados bilaterales, la Convención establece que se considerarán en vigor cuando así lo acuerden expresamente las partes interesadas, esto es, el Estado de reciente independencia y el Estado parte no afectado por la sucesión de Estados, o cuando éstas se comporten de forma que deba entenderse que han convenido en ello.

La práctica de los Estados de reciente independencia se ha desarrollado mayoritariamente en el sentido de las disposiciones de la Convención de 1978, no en vano hay que recordar que el proceso de descolonización se encontraba muy avanzado en el momento de la celebración de la Conferencia de codificación y que la CDI había mostrado una atención preferencial hacia esos casos. No obstante, debe señalarse una variante de la tabla rasa en forma de contracting-out, conocida con el nombre de doctrina Nyerere, la cual consideraba que los Estados de reciente independencia podían decidir qué tratados no les eran aplicables durante un período de tiempo en el que, sobre la base de la reciprocidad, dichos tratados podían considerarse en vigor, si bien transcurrido dicho período los tratados que no hubieran sido objeto de ninguna actuación decaían. En cualquier caso, habría que destacar que la posibilidad de elegir qué tratados continúan en vigor, de una u otra forma, es prácticamente una constante en los Estados surgidos del proceso descolonizador $\mathrm{y}$, aunque dicho proceso se encuentra abocado al agotamiento, todavía pueden citarse dos 
ejemplos recientes: Namibia y los Estados federados de Micronesia.

La última categoría incluida en la Convención de Viena sobre sucesión de Estados en materia de tratados es la de separación de partes de un Estado y se refiere al hecho de que una parte o partes del territorio de un Estado se separen para formar uno o varios Estados, continúe o no existiendo el Estado predecesor. Para estos casos, la Convención establece un régimen inspirado en el principio de continuidad automática, es decir, los tratados del predecesor seguirán en vigor respecto del sucesor, salvo que los Estados interesados convengan otra cosa o que resulte incompatible con el objeto y el fin del tratado.

Sin embargo, los acontecimientos de los últimos años han mostrado la contradicción de las previsiones de la Convención de 1978 con la práctica de los nuevos Estados surgidos por esa vía. Una atenta aproximación a dicha práctica permitiría descomponer esta categoría atendiendo a cómo se produce la sucesión de Estados, esto es, a si es el resultado de un proceso progresivo, pacífico o pactado, lo que hemos llamado disolución de Estados, o si es la consecuencia de una serie de acontecimientos traumáticos, en los que pueden concurrir elementos de violencia, lo que hemos denominado desmembramiento de Estados. ${ }^{14}$

En el primer caso, la disolución de Estados, el modo en que tiene lugar la sucesión hace

${ }^{14} \mathrm{La}$ abundante práctica internacional reciente en relación con el conjunto de cuestiones que se plantean en estos tipos sucesorios es analizada con mayor profundidad en ORTEGA TEROL, J.M., El desmembramiento de Estados en la Europa de fin de siglo, ed. Tirant Lo Blanch, Valencia, 1999. que la mayor parte de las cuestiones relativas a la misma sean abordadas conjuntamente por los Estados implicados, incluso con anterioridad a alcanzar la plena subjetividad internacional, inclinándose claramente por la continuidad de los vínculos contraidos por el Estado predecesor. Un ejemplo en este sentido puede ser el de la disolución de Checoslovaquia, cuyos Estados resultantes, Repúblicas Checa y Eslovaca, manifestaron prontamente su adscripción a los principios contenidos en la Convención de Viena al respecto.

Por el contrario, en los casos de desmembramiento de Estados, los hechos de que trae causa la sucesión de Estados implican que los nuevos Estados cuestionen, en gran medida, su continuidad respecto de los vínculos contraidos por el predecesor. La práctica más reciente muestra cómo estos Estados han hecho uso de la técnica de la notificación de sucesión, alternativamente con otros modos de manifestación del consentimiento en obligarse por tratados internacionales, en una línea muy similar a la seguida por los Estados de reciente independencia, poniendo de manifiesto una clara tendencia hacia el principio de la tabla rasa matizada. En esta dirección, pueden mencionarse los ejemplos de las Repúblicas resultantes del desmembramiento de la URSS $^{15}$ - incluyendo tratados sobre derechos humanos, lo que llamó la atención de algunos órganos de control de los mismos, como el

${ }^{15}$ Vid. Memorándum relativo al consenso sobre la cuestión de la sucesión de Estados en relación con los tratados de la ex-URSS que presentan un interés mutuo; ORTEGA TEROL, J.M., Textos y Documentos sobre los desmembramientos de la Unión Soviética y de Yugoslavia, Ed. UCLM, Cuenca, 1996, p. 60. 
Comité contra la eliminación de todas las formas de discriminación racial - $\mathrm{o}$, de forma más evidente, el caso de Yugoslavia ${ }^{16}$.

En relación con los tratados bilaterales la solución dada en estos supuestos, a favor de una elevada capacidad de opción, resulta más evidente, como puede deducirse por ejemplo de los acuerdos celebrados por las repúblicas ex-yugoslavas con Austria, o por citar el ejemplo más reciente que nos afecta del acuerdo bilateral entre BosniaHerzegovina y España (de 21 de enero de 2004) que, aparentemente, reposaría en la idea de continuidad, pero no tiene reparos en no considerar vigente el Acuerdo de Transporte Aéreo, de 11 de abril de 1979, algo que sólo cabría esperar si se toma como punto de partida la tabla rasa.

Todavía en este apartado habría que hacer referencia a dos cuestiones que afectan a todos los supuestos aquí considerados y que tienen que ver con determinados tipos de tratados. En primer lugar, hay que señalar que, con carácter general, se excluye la continuidad de la condición de miembro de una Organización internacional en la figura de los Estados sucesores, con la excepción de los supuestos de transferencia territorial, que deberán seguir las reglas establecidas en relación con la adquisición de aquella condición. Dicho con otras palabras, los Estados sucesores, ya sean resultado del proceso de descolonización, ya lo sean de un desmembramiento o una disolución de

${ }^{16}$ Vid. Auto de la CIJ, de 8 de abril de 1993, asunto sobre la aplicación del Convenio sobre prevención y sanción del delito de genocidio, Bosnia-Herzegovina contra Yugoslavia (Serbia y Montenegro), y la Sentencia, de 11 de julio de 1996, sobre las excepciones preliminares en el mismo asunto.
Estados, deberán solicitar su admisión como nuevos miembros.

No obstante, al menos en lo que respecta a la práctica de la ONU, los casos de unificación muestran soluciones diversas. Así, cuando los Estados que se unen son individualmente miembros de la Organización, parece bastar una nota comunicando la nueva situación a dicha organización y pasar a ser un único miembro. Esto es lo que ocurrió con la formación de la República Arabe Unida, resultado de la unión de Egipto y Siria, en 1958, con la constitución de Tanzania por parte de Tanganyika y Zanzibar, en 1964, o, más recientemente, con Yemen. ${ }^{17}$

Finalmente, la segunda cuestión a la que hacíamos referencia tiene que ver con algo que ya ha sido señalado anteriormente: la necesidad de que los Estados sucesores respeten las fronteras internacionales preexistentes, así como otros regímenes territoriales. En este sentido, la jurisprudencia de la CIJ se inclina por considerar el carácter declarativo de las reglas contenidas en los artículos 11 y 12 de la Convención de Viena de 1978, que establecen la continuidad de unas y otros independientemente de la forma en que se produzca la sucesión de Estados.

\subsubsection{Sucesión de Estados y responsabilidad internacional}

El principio general en relación con esta cuestión es que la responsabilidad internacional derivada de actos ilícitos del Estado predecesor no se transmite a los Estados sucesores, esto es, ningún Estado

${ }^{17}$ ANDRES SAENZ DE SANTAMARIA, M.P., op. cit., pp. 167-168. 
sucesor está obligado a reparar los daños causados por su predecesor, solamente el autor del acto es responsable. ${ }^{18}$

\subsection{Sucesión de Estados en materia de bienes, archivos y deudas de Estado}

Si decíamos que la Convención sobre sucesión de Estados en materia de tratados internacionales planteaba problemas, aún se puede hacer esa afirmación con mayor rotundidad en relación con la Convención sobre sucesión de Estados en materia de bienes, archivos y deudas de Estado, algo que, teniendo en cuenta cómo puede producirse dicha sucesión, resulta perfectamente explicable cuando de lo que se trata es de dividir el activo y el pasivo de un Estado. Efectivamente, aquí el interrogante versaría sobre qué bienes y deudas pasan a ser del Estado sucesor. Por ello, tal vez adquieran mayor relevancia las diferencias entre supuestos donde la sucesión se produce pacíficamente y aquellos otros en que existen serias divergencias entre los Estados sucesores.

No obstante, habría que destacar que, si bien la Convención de Viena de 1983, al igual que su predecesora de 1978, contempla soluciones distintas para supuestos diversos, en este caso puede observarse la existencia de un principio general, excepto en los supuestos de unificación y de Estados de reciente independencia: la división de las propiedades y las deudas del Estado predecesor puede hacerse mediante acuerdo que suponga un reparto equitativo. Únicamente en el

\footnotetext{
${ }^{18}$ Tribunal arbitral franco-helénico, asunto de los faros, laudo de 24 de julio de 1956, RSA, vol. XII, p. 161.
}

caso de que tal acuerdo no sea posible, se establece una serie de reglas subsidiarias aplicables de forma diferenciada, en una regulación extremadamente compleja y que ha demostrado una escasa eficacia en la solución de los problemas para los que fue diseñada. La cuestión todavía se complica más si se tiene en cuenta que el acuerdo entre los Estados sucesores no vincula a terceros Estados interesados en la sucesión, como es el caso de acreedores del Estado predecesor.

Por una parte, la Convención aborda separadamente cada uno de los ámbitos a que se refiere, esto es, bienes, archivos y deudas. Se ha señalado acertadamente que la distinción entre bienes y archivos puede parecer superflua, toda vez que los archivos son una clase de bienes. Sin embargo, la justificación de esa diferencia vendría dada por el hecho de que éstos son, en principio y con los mecanismos técnicos actuales, fácilmente reproducibles, lo que permitiría soluciones distintas a las pergeñadas para otros bienes. ${ }^{19}$ Asimismo, las definiciones dadas a cada una de esas categorías no están exentas de dificultades como demuestra el ejemplo de Yugoslavia, donde, entre otras cosas, la definición de bienes de Estado se ha erigido en un obstáculo para la consecución de un acuerdo.

Desde el punto de vista práctico, algunos supuestos no plantean demasiados problemas. Así, en los casos de unificación de Estados o de absorción de un Estado por otro. El hecho de que estos acontecimientos sólo puedan producirse de forma pacífica, unido a que se trata de una cuestión de acumulación,

${ }^{19}$ REMIRO et al., Derecho Internacional, Madrid, McGraw-Hill, 1997, p. 65. 
no de división, de bienes y deudas facilita enormemente la tarea de alcanzar una rápida conclusión respecto del destino final de ambos tras una sucesión de Estados. Como fácilmente habrá adivinado el atento lector, la solución es el paso de bienes, archivos y deudas de los Estados predecesores al Estado sucesor $^{20}$. En este sentido, y en consonancia con lo dispuesto en la Convención de 1983, parece ir la práctica de los Estados.

Más controvertidas resultan las disposiciones relativas a los Estados de reciente independencia. En estos supuestos, frente a la regla general del acuerdo para la solución de los problemas planteados y como excepción a la misma, la Convención establece una serie de reglas directamente aplicables a la aparición de nuevos Estados como consecuencia de la descolonización que suponía un régimen enormemente favorable para aquellos, asumiendo los postulados de los Estados en vías de desarrollo surgidos de la descolonización en el contexto de una oposición frontal a las estructuras económicas existentes. No obstante, algunas de las soluciones aportadas tienen un alcance que excedería al del estricto supuesto de Estados de reciente independencia, como es el principio locus in quo, es decir, los bienes situados en el territorio del Estado sucesor pasan a éste y ello, en principio, sin compensación. Por lo demás, se trata de una regulación prolija, de la que cabe destacar la ausencia de un deber de asumir deudas del Estado predecesor por los Estados de reciente independencia, a menos que medie

\footnotetext{
${ }^{20}$ Arts. 16, 29 y 39 de la Convención de Viena sobre sucesión de Estados en materia de bienes, archivos y deudas de Estado.
}

un acuerdo en sentido contrario en razón del nexo entre la deuda y el territorio de éstos.

Actualmente, sin embargo, lo más destacable es el contraste que han sufrido las disposiciones relativas a las categorías de separación de parte o partes del territorio de un Estado y de disolución de un Estado frente a los acontecimientos siguientes a la caida del muro de Berlín, poniendo de manifiesto la ineficacia de las mismas. En una primera aproximación, habría que recordar que la distinción entre ambas categorías reposa en la continuidad en la identidad o no del Estado predecesor, por lo que cabe reproducir aquí las consideraciones realizadas acerca de la inadecuación de una tipología sucesoria que incluya dicha continuidad como elemento diferenciador. Incluso puede observarse cómo la clasificación establecida en el Convenio de 1983 ha supuesto un obstáculo importante en la disputa jurídica acerca de la distribución de los bienes, archivos y deudas de la antigua Yugoslavia, dadas las divergentes posturas de las partes sobre la existencia de aquel elemento.

La Convención de 1983, como hemos mencionado, establece la prevalencia del acuerdo entre las partes para la solución de las diversas cuestiones planteadas. A falta de tal, en supuestos de disolución con desaparición del predecesor y en relación con los bienes, se prevé el paso de los bienes inmuebles al Estado en cuyo territorio se encuentren, el reparto equitativo de los bienes inmuebles situados fuera del territorio del Estado predecesor, el paso de los bienes muebles al Estado a cuyo territorio se encontraran vinculados en razón de la actividad desarrollada por el predecesor y la distribución en proporciones equitativas del resto de bienes muebles. $\mathrm{La}$ 
diferencia existente con la regulación prevista para los supuestos de separación estriba en que, en este caso, nada se dice de los bienes inmuebles situados en el extranjero. En relación con los archivos, la ausencia de acuerdo se traduciría básicamente en el paso al Estado sucesor de aquellos archivos que tengan una conexión territorial con el mismo. La principal divergencia entre los supuestos a los que nos venimos refiriendo se encontraría en que se permite un mayor control al Estado predecesor, en caso de que continuara su existencia, sobre los archivos contemplados. Por último, la redacción de las disposiciones relativas a las deudas es idéntica en ambos casos: sin acuerdo pasará al Estado sucesor una proporción equitativa de la deuda del predecesor, teniendo en cuenta en particular los bienes, derechos e intereses que pasen a aquél en relación con esa deuda.

Con ese punto de partida, puede decirse que los datos de la práctica estatal más reciente no hacen sino subrayar la importancia del acuerdo entre las partes implicadas en la solución de los problemas a este respecto. En este sentido, como resulta obvio, no parece haber sido difícil alcanzar tal resultado en un caso de disolución pacífica como es el de la República checoslovaca. ${ }^{21}$ Aquí, previamente a la disolución del Estado predecesor, el 31 de diciembre de 1992, se adoptó una Ley sobre división de las propiedades federales, que establecía un criterio territorial para la división de los bienes localizables (principio

${ }^{21}$ Como ejemplo al respecto, pueden resultar de interés las consideraciones vertidas por el Abogado General del Tribunal de Luxemburgo, Antonio TIZZANO, en sus conclusiones en el Asunto C-216/01, Budejovický Budvar, národni podnik, contra Rudolf Ammersin GMBH, de 22 de mayo de 2003. locus in quo), un criterio proporcional para la distribución del resto de bienes y la deuda externa, incluyendo las fuerzas armadas y las reservas federales, atendiendo a la ratio de población de las Repúblicas constitutivas (2 partes para Chequia y 1 para Eslovaquia), y se preveían también algunos supuestos de compensación. ${ }^{22}$

Más complejo ha resultado el proceso en el desmembramiento soviético. ${ }^{23}$ La primera cuestión en ser abordada fue el reparto de la deuda soviética, estimada en más de 60.000 millones de dólares, y se llegó a un acuerdo, en diciembre de 1991, sobre las cuotas de división de la misma, aunque se establecía el principio de responsabilidad solidaria de todas las Repúblicas. Paralelamente, se pergeñaba la solución respecto de los bienes de la URSS en el extranjero, cuestión que también había sido incluida en el acuerdo anterior, pero que fue objeto de mayor precisión en un acuerdo de 6 de julio de 1992, ${ }^{24}$ donde se establecía la cuota de participación de los Estados miembros de la Comunidad de Estados Independientes. Mientras, para los bienes situados dentro de la antigua Unión Soviética se había seguido la

${ }^{22}$ ANDRES SAENZ DE SANTAMARIA, op. cit., p.197; KOSKENNIEMI, M., «The present state of research», en State succession: codification tested against the facts, Academia de Derecho Internacional de La Haya, 1996, p. 120.

\footnotetext{
${ }^{23}$ Junto al trabajo del Profesor BLANC ALTEMIR citado anteriormente (ver nota 7), puede citarse, entre nosotros, el reciente estudio de Tatsiana USHAKOVA, La sucesión de Estados en materia de bienes, archivos y deudas: el caso de la URSS, Madrid, Ed. Ramón Areces, 2006.

${ }^{24}$ Vid. ORTEGA TEROL, J.M., Textos...., op.cit., pp. 63-66.
} 
regla locus in quo. Por otra parte, en aquella misma fecha, se acordaba el destino de los archivos de Estado de la ex-URSS. ${ }^{25}$

Sin embargo, existieron grandes dificultades para la aplicación de algunos de esos acuerdos. Así, en octubre de 1992, en la reunión de la CEI celebrada en Bishkek, se decidía finalmente resolver la cuestión de las deudas junto con la división de los bienes de forma bilateral. Esta solución, en gran medida, fue dictada por la preferencia de los acreedores occidentales de tratar con un solo deudor. La Federación Rusa se mostró dispuesta a atribuirse ese papel siempre que se hiciera sobre la base de un intercambio: asumir la deuda atribuida a cualquiera de las Repúblicas contra su parte en los bienes soviéticos en el extranjero. En esta dirección, a lo largo de 1992 y 1993 se concluyeron acuerdos bilaterales entre los Estados sucesores y Rusia.

No obstante, esta forma de solucionar la cuestión no satisfizo a Ucrania, debido fundamentalmente a la ausencia de acuerdo sobre el control de la flota del Mar Negro. La posición ucraniana se tradujo en una petición a la Comunidad Internacional de conservar y no alterar el status de la propiedad de la antigua Unión Soviética en el extranjero hasta que no se alcanzase una solución definitiva. El problema, agravado por el mantenimiento de una fuerte presencia militar rusa en la península de Crimea, con tintes de pretensión territorial, parece haber obtenido esa solución con el Tratado de Cooperación y Amistad, de 31 de mayo de 1997, por el que Rusia reconoce la soberanía ucraniana en esa

\footnotetext{
${ }^{25}$ Ibid., pp. 66-68.
}

zona y Ucrania alquila a Rusia una serie de infraestructuras por un período de 20 años.

La solicitud realizada por Ucrania proporciona un dato relevante sobre la situación que se produce ante la falta de acuerdo, algo que resulta todavía más evidente en el caso yugoslavo. Efectivamente, las discrepancias entre las Repúblicas ex-yugoslavas en la calificación jurídica del supuesto sucesorio se tradujeron en una ausencia de acuerdo desde el inicio. La Conferencia para la Paz en la antigua Yugoslavia estableció un Grupo de Trabajo sobre cuestiones de sucesión que trató de encontrar una solución, desde los primeros momentos de la crisis yugoslava. Asimismo, la Comisión de Arbitraje dedicó su atención a los problemas de sucesión en materia de bienes, archivos y deudas. ${ }^{26}$ No obstante, el impasse duró cerca de diez años en los que la situación fue provisional, generando en ocasiones fuertes disputas acerca de a quién cabía imputar la titularidad de tal o cual bien, fundamentalmente depósitos bancarios. Y, sin embargo, todos esos esfuerzos encontraron recompensa hasta el 29 de junio de 2001, cuando se firmó el Acuerdo sobre cuestiones sucesorias entre los cinco Estados sucesores de la antigua Yugoslavia. Dicho acuerdo constituye un complejo entramado, con siete anexos, que aborda los problemas derivados de la sucesión en el ámbito de los

\footnotetext{
${ }^{26}$ En primer lugar, en su Dictamen n. ${ }^{\circ} 9$, de 4 de julio de 1992, había insistido en que todos los aspectos de la sucesión debían solucionarse mediante acuerdo. En segundo lugar, más detenidamente, los Dictámenes $12,13,14$ y 15 versan sobre diversos problemas de sucesión en materia de bienes, archivos y deudas. Pueden encontrarse en ORTEGA TEROL, J.M., Textos...., op. cit., pp. 94-95 y 101-109.
} 
bienes, archivos y deudas de Estado. ${ }^{27}$ Este viene a poner de manifiesto que, en esta materia, ante la falta de consenso entre las partes implicadas, y en ausencia de cauces institucionales que impongan una solución determinada, lo único claro es la aplicación del principio locus in quo, tanto para los bienes como para las deudas. Mientras, los Estados sucesores pueden solicitar, y el resto de Estados adoptar, las medidas que consideren adecuadas para la protección de sus respectivos intereses.

\subsection{La sucesión de Estados y su impacto sobre la nacionalidad de los habitantes del territorio}

La última cuestión que vamos a abordar en relación con las transformaciones territoriales de los Estados es la de su influencia sobre la nacionalidad de los habitantes de un territorio. Como resulta obvio, el nacimiento de un nuevo Estado, la desaparición de uno existente, la transferencia de un territorio de un Estado a otro son fenómenos que pueden tener incidencia sobre la nacionalidad de los habitantes de los territorios afectados por dichas alteraciones, en el sentido de que hay cambios en la nacionalidad. Debe señalarse que la CDI ha elaborado un texto articulado sobre esta cuestión y, dadas las dificultades encontradas por las dos Convenciones sobre sucesión de Estados, la forma final ha sido la de una declaración de la AG, sin que se plantee actualmente la convocatoria de una conferencia de plenipotenciarios con vistas a

${ }^{27}$ Un análisis pormenorizado del acuerdo puede verse en ORTEGA TEROL, J.M., "La sucesión...", op. cit., pp. 137-142. su conversión en tratado, subrayando quizás de esta forma las enormes dificultades que entraña abordar este tipo de cuestiones ${ }^{28}$.

No obstante, puede observarse que la práctica estatal al respecto está lejos de ser uniforme y de concretar una solución unívoca a esta cuestión, aunque conviene subrayar que sobre la globalidad de los problemas que se derivan de una sucesión de Estados sobre la nacionalidad de los habitantes de un territorio planea siempre la necesidad de evitar la apatridia.

Así, en casos de unificación o de absorción de un Estado por otro, lo normal es que se extienda la nueva nacionalidad a todos los que poseían la nacionalidad de los Estados predecesores. Por tanto, el problema se centra en los supuestos de aparición de nuevos Estados y en los casos de transferencias territoriales. Las posibles soluciones irían en dos direcciones: la adquisición automática de la nacionalidad del Estado sucesor y el ofrecimiento de una opción por la nacionalidad del Estado predecesor, en el caso de que ésta subsista, o por la nacionalidad de los diversos Estados sucesores que resulten de la sucesión de Estados. Esta opción puede articularse, bien por vía convencional, a través de un tratado internacional, bien por la ley interna de los Estados implicados en la sucesión.

La cuestión puede adquirir una gran complejidad. Por una parte, en el caso de que el Estado predecesor continúe existiendo o haya un Estado que se pretenda

\footnotetext{
${ }^{28}$ Una perspectiva detallada sobre la problemática expuesta puede encontrarse en TORRES CAZORLA, M.I., La sucesión de Estados y sus efectos sobre la nacionalidad de las personas físicas, Universidad de Málaga, 2001.
} 
continuador de aquél, teniendo en cuenta que la determinación de la nacionalidad de los habitantes de un Estado es una cuestión esencialmente dependiente de su derecho interno, lo habitual es que mantenga ese vínculo jurídico respecto de sus nacionales, produciéndose de esta forma supuestos de múltiple nacionalidad. Por otra parte, el ejercicio del derecho de opción puede llevar implícita la obligación de trasladar la residencia al Estado por cuya nacionalidad se opta. Tras la Primera Guerra Mundial, la inclusión de esa obligación en la mayoría de los Tratados de Paz dio lugar a la vergonzosa práctica de las transferencias de población.

Finalmente, debe hacerse una mención separada en este punto a la reaparición de los Estados bálticos. En efecto, la tesis sostenida por Letonia, Estonia y Lituania, ha supuesto que, por ejemplo, los dos primeros «resuciten» las leyes sobre nacionalidad vigentes en 1940, restringiendo enormemente el acceso a la misma de la población rusoparlante y provocando con ello una delicada situación.

\section{A MODO DE CONCLUSIÓN: ¿SUCESIÓN DE ESTADOSO PROCESOS SUCESORIOS?}

El derrumbamiento del bloque soviético que ha llevado aparejado el nacimiento de nuevos Estados en la Europa del Este ha puesto a prueba las concepciones más tradicionales de la institución de la sucesión de Estados y, muy particularmente, las vertidas en las Convenciones de Viena en la materia. La práctica más reciente ha mostrado la enorme confusión que se produce a la hora de valorar, en primer lugar, si existe continuidad en la identidad del Estado afectado por una transformación territorial, en la que parece jugar un papel preponderante el reconocimiento de las pretensión de continuidad por los Estados directamente resultantes del proceso de transformación y por la comunidad internacional en su conjunto. En segundo lugar, y como consecuencia de ello, las categorías sucesorias incluidas en las Convenciones de Viena resultan frecuentemente inservibles en los supuestos en que la sucesión se produce de una forma traumática, lo que nosotros hemos denominado desmembramiento de Estados, dada la disparidad de criterios entre las entidades estatales resultantes. Por el contrario, transcurridos más de quince años desde el inicio de los acontecimientos, puede apreciarse que la solución de los problemas que conllevan suele articularse como un proceso, cuyos resultados dependen básicamente del acuerdo entre las partes directamente afectadas, con la ocasional participación de terceros implicados, especialmente en lo que se refiere a bienes y deudas. Las reglas de los Convenios con pretensiones de codificación - y fundamentalmente del Convenio de 1983 -, lejos de asegurar un resultado predeterminado, apenas han pasado de ser criterios orientativos que, en algún caso, como en los supuestos de la Unión Soviética y de Yugoslavia han servido más bien para enquistar las posturas negociadoras de los Estados que se pretendían continuadores. Dicho de otra forma, no parece que el Derecho internacional, más allá de algunas pocas reglas bien asentadas, como las que se refieren a la estabilidad de las fronteras o algunos regímenes territoriales, proporcione soluciones susceptibles de ser aplicadas 
inmediatamente a las transformaciones territoriales, sino que éstas se articulan en procesos que los Estados implicados van desarrollando según sus propios intereses.

Así, por lo que se refiere a los aspectos que han sido objeto de codificación por la CDI, los Estados pueden decantarse por la aplicación de la regla de la continuidad de los tratados internacionales si estiman que ello puede dar un mayor impulso a su situación relativa en la escena internacional, mientras que si las líneas de fricción entre los Estados implicados en una situación sucesoria son especialmente afiladas tenderán a adoptar posiciones más próximas a la regla de la tabla rasa. En una y otra circunstancia puede, incluso, que la solución finalmente adoptada tarde mucho tiempo en cristalizar, como se ha puesto de manifiesto en el caso checoslovaco y sus relaciones con Austria, o como ha sucedido en el caso español con Eslovenia, en los que han pasado más de diez años hasta dar con soluciones que lo mismo podrían argumentarse desde un punto de vista, que desde el contrario. Otro tanto ocurre en relación con el reparto de bienes, archivos y deudas, en el que la posibilidad de que un Estado pueda prevalerse de la posición privilegiada que supone erigirse en continuador en la identidad del predecesor puede generar no pocas susceptibilidades entre los demás Estados implicados. Sobre todo en este último supuesto, como ha podido verse de forma particular en el supuesto yugoslavo, la idea de proceso tiene una mayor fuerza: hasta el 29 de junio de 2001 no se firmó el Acuerdo sobre cuestiones sucesorias entre los cinco Estados sucesores de la exYugoslavia. 